\title{
Highly sensitive chromatographic time-resolved fluoroimmunoassay for rapid onsite detection of streptomycin in milk
}

\author{
Zile Wang, ${ }^{1 *}$ ๑ Yuanze Sun, ${ }^{2 *} \odot$ Demei Liang, ${ }^{1} \odot$ Yuyang Zeng, ${ }^{1} \odot$ Shuang He, ${ }^{1} \odot$ Ghulam Mujtaba Mari, ${ }^{1}$ \\ Tao Peng, ${ }^{1}$ (i) and Haiyang Jiang ${ }^{1} \dagger$ (i) \\ ${ }^{1}$ Beijing Advanced Innovation Center for Food Nutrition and Human Health, College of Veterinary Medicine, China Agricultural University, \\ Beijing Key Laboratory of Detection Technology for Animal-Derived Food Safety, Beijing Laboratory for Food Quality and Safety, \\ 100193 Beijing, People's Republic of China \\ ${ }^{2}$ Shunyi Animal Health Supervision and Administration Bureau, Beijing 101300, People's Republic of China
}

\section{ABSTRACT}

Antibiotic residues are major contaminants in milk because of their use in agriculture and animal husbandry. In particular, streptomycin, an aminoglycoside antibiotic, is a potential risk to consumers because of its ototoxicity, anaphylaxis, and growth inhibition. Herein, monoclonal antibodies for streptomycin were conjugated with europium microspheres to serve as detection probes for the development of a chromatographic time-resolved fluoroimmunoassay to detect streptomycin residues in milk. The method had a low detection limit of $0.58 \mu \mathrm{g} / \mathrm{kg}$, a linear range of 0.8 to $6.25 \mu \mathrm{g} / \mathrm{kg}$, and substantial recovery, from 85.6 to $108.3 \%$. It showed slight cross-reactivity with another aminoglycoside analog. Strong correlations between the results of established chromatographic time-resolved fluoroimmunoassay and ultra-performance liquid chromatography-tandem mass spectrometry indicated that the established fluoroimmunoassay is a reliable method for rapid onsite detection of streptomycin in milk and it has great potential in food safety monitoring.

Key words: chromatographic time-resolved fluoroimmunoassay, streptomycin, residue detection, milk

\section{INTRODUCTION}

Under the influence of economic globalization, standards of living have improved and global demand for milk and other dairy products has increased dramatically (Chen et al., 2018). Therefore, the prevention of dairy cow diseases, such as mastitis, is very important to ensure a safe milk supply and reduce the economic

Received February 19, 2020.

Accepted May 6, 2020.

*These authors contributed equally to this work.

$\dagger$ Corresponding author: haiyang@cau.edu.cn losses of the breeders. Streptomycin (STR; Figure 1), an aminoglycoside antibiotic, is widely used as a feed additive and agent in dairy cow husbandry due to its excellent performance in treating mastitis, a disease caused by gram-negative bacteria and Mycobacterium tuberculosis (Zhang et al., 2018). Although streptomycin is effective in preventing and treating mastitis, its ototoxicity and anaphylaxis cannot be ignored (Wang et al., 2017b). Widespread use without supervision can lead to streptomycin residues in milk and throughout the food chain, endangering human health. To effectively monitor streptomycin residues and protect human health, regulatory authorities have established maximum residue limits for streptomycin in food. In the European Union, the maximum residue limit for streptomycin in bovine and ovine milk is $200 \mu \mathrm{g} / \mathrm{kg}$, and other countries have similar regulations (Zhao et al., 2017).

At present, the methods available for detection of streptomycin residues in milk can be divided into microbiological assays, instrumental assays, and immunoassays (Chafer-Pericas et al., 2010). As the requirements for residue detection include speed and portability, these methods have some obvious shortcomings. Microbiological assays require bacterial culture, they are time consuming, and the results are affected by many factors that can lead to poor sensitivity and specificity. Instrumental methods not only require expensive detection equipment and complicated sample pretreatment procedures but also have higher professional requirements for experimental operators. Current methods cannot be applied to rapid screening of large quantities of samples in the field. Immunoassays, including ELISA (Wu et al., 2010; Xi et al., 2013), colloidal gold immunochromatography (GICA; Verheijen et al., 2000), time-resolved fluoroimmunoassay (TRFIA; Sun et al., 2017), chemiluminescence immunoassay (CLIA; Tao et al., 2017) and chemiluminescent enzyme immunoassay (CLEIA; Yu et al., 2014; Zeng et al., 2016), have reduced requirements for operators and shortened detec- 
Wang et al.: RAPID IMMUNOASSAY TO DETECT STREPTOMYCIN IN MILK

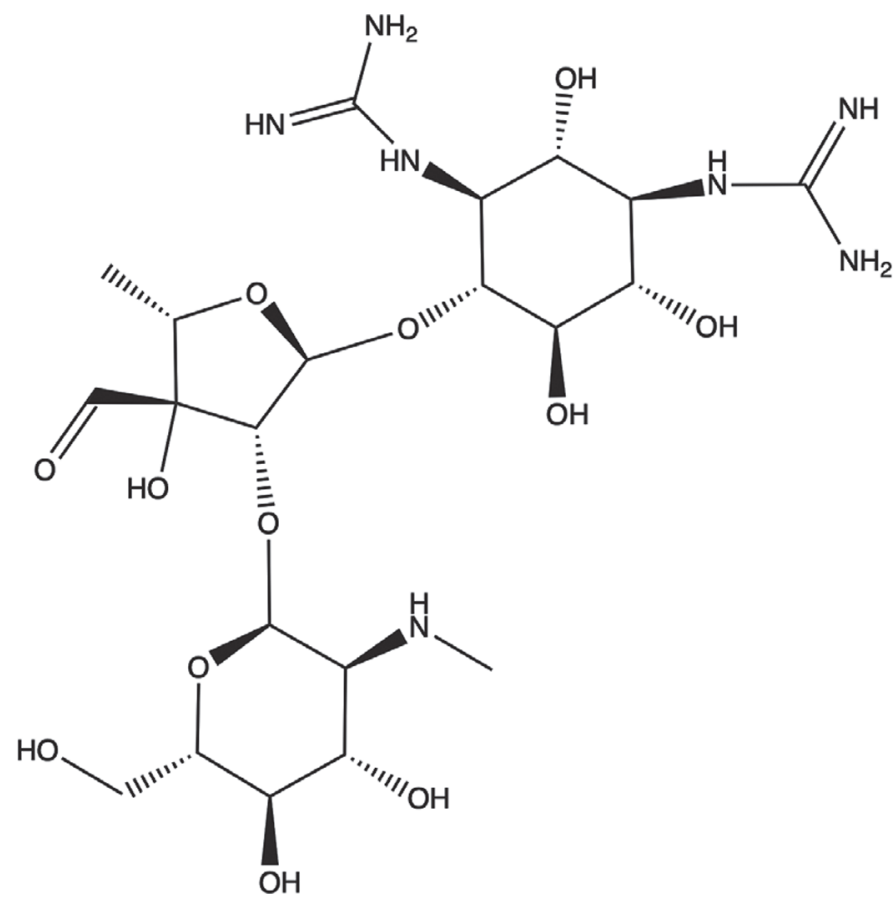

Figure 1. Chemical structure of streptomycin.

tion times, but they still have complicated incubation steps making real-time detection difficult. Thus, these approaches cannot fulfill the need for fast, simple, onsite detection of streptomycin residues in milk.

Antibodies and labeling materials are the basis of immunological detection methods. In recent years, nanomaterials have been applied in the development of immunoassay methods, such as gold nanoparticles (Peng et al., 2014), latex beads (Wang et al., 2017a), and carbon nanoparticles (Posthuma-Trumpie et al., 2012), to function as visual reporters, but they can only achieve qualitative or semi-quantitative detection because of light interference and the need for subjective judgment (Huang et al., 2016). Other methods, including superparamagnetic beads (Zhang et al., 2013), quantum dots (Taranova et al., 2015), and upconversion phosphors (Wang et al., 2016), can achieve quantitative detection via reflectance and fluorescence readers, but the complex matrices may interfere with the immunoreaction and lead to ambiguous results (Juntunen et al., 2012). Lanthanides such as europium $(\mathrm{Eu})(\mathrm{III})$, terbium (Tb)(III), samarium (Sm)(III), and dysprosium (Dy)(III) have attracted attention because of their long fluorescence lifetime and large Stokes shift, which is beneficial in reducing nonspecific interference of background fluorescence signals and thus improving sensitivity (Buenzli, 2010; Hagan and Zuchner, 2011). According to reports, lanthanide chelate nanoparticle labels have been applied for the detection of drugs $(\mathrm{Hu}$ et al., 2017), toxins (Wang et al., 2015, Zhang et al., 2015), bacteria (Cho et al., 2015), and proteins (Soukka et al., 2003).

In this study, we coupled $\mathrm{Eu}(\mathrm{III})$ nanoparticles with anti-streptomycin antibodies to serve as probes, and we established a chromatographic time-resolved fluoroimmunoassay (CTRFIA) that used immunochromatography to detect streptomycin residues in milk.

\section{MATERIALS AND METHODS}

\section{Materials and Reagents}

Streptomycin, gentamycin, spectinomycin, kanamycin, tobramycin, amikacin, and dihydrostreptomycin were purchased from Sigma-Aldrich (St. Louis, MO). Goat anti-mouse antibody, anti-streptomycin mAb (anti-STR mAb), and streptomycin-ovalbumin (STROVA) were previously prepared in our laboratory. The 1\% (solid content, wt/vol) $\mathrm{Eu}(\mathrm{III})$-marked and $\mathrm{COOH}$-modified monodisperse polystyrene beads were purchased from Weice Biotechnology Co. Ltd. (Shanghai, China). 1-Ethyl-3-(3-dimethylaminopropyl)-carbodiimide (EDC), hydroxysuccinimide (NHS), and morpholinoethanesulfonic acid (MES) were purchased from Aladdin Chemistry Company (Shanghai, China). The nitrocellulose (NC) membrane, absorbance pads, and sample pads were purchased from Shanghai Jingbiao Biotechnology Company (Shanghai, China). All the other chemicals and solvents were of analytical grade and obtained from Beijing Chemical Reagent Co. Ltd. (Beijing, China).

The XYZ dispensing platform and cutting machine were supplied by Shanghai Jinbiao Biological Company (Shanghai, China). The digital blast drying oven was purchased from Yuanheng Oven Equipment Co. Ltd. (Wujiang, China). The fluorescent reader was purchased from Nanjing Weice Biotechnology Co., Ltd. (Nanjing, China). Deionized water was obtained using a Milli-Q system from Millipore Corp. (Bedford, MA).

\section{Characterization of Europium Microspheres}

Before the preparation of fluorescent probes, a solution of europium microspheres was diluted 100 times by using $0.01 \mathrm{~mol} / \mathrm{L} \mathrm{PBS}$ at $\mathrm{pH} 7.4$ and then subjected to UV scanning in the range of $250-750 \mathrm{~nm}$ using an UV spectrophotometer to obtain an UV absorption spectrum for europium microspheres. Then, the europium microspheres were diluted 10,000 times by using $0.01 \mathrm{~mol} / \mathrm{L}$ PBS solution at $\mathrm{pH} 7.4$ and characterized by multifunctional microplate reader SpectraMax M5 (Molecular Devices LLC, San Jose, CA) to obtain the fluorescence spectrum. 
In addition, hydrochloric acid and sodium hydroxide were used to adjust the $\mathrm{pH}$ of $0.01 \mathrm{~mol} / \mathrm{L}$ PBS to obtain buffer solutions with $\mathrm{pH}$ of $5,6,7,8$, and 9 ; buffer solutions with $0,0.1,0.5$ and $1 \mathrm{M} \mathrm{NaCl}$ were prepared at the same time. Then, europium microspheres were diluted 10,000 times with the above solutions, respectively, and we determined the effects of solutions with different $\mathrm{pH}$ and salt ion concentrations on the optical stability of europium microspheres by measuring fluorescence intensity.

\section{Preparation of Europium Microsphere- Antibody Conjugates}

The fluorescent probes were obtained by covalently coupling carboxylate-modified europium microspheres with anti-STR antibodies via an active ester method. Briefly, EDC and NHS were added to $1 \mathrm{~mL}$ of MES buffer, which contained $10 \mu \mathrm{L}$ of europium microsphere solution, and the mixture was stirred at room temperature for $15 \mathrm{~min}$. Subsequently, the activated nanoparticles were separated by centrifugation at $10,000 \times g$, $4^{\circ} \mathrm{C}$, for $10 \mathrm{~min}$, and the precipitate was resuspended in $1 \mathrm{~mL}$ of coupling buffer and evenly dispersed by sonication for $1 \mathrm{~min}$. Thereafter, anti-STR antibodies were added to the microspheres and gently mixed for $2 \mathrm{~h}$ at room temperature. After centrifugation at $10,000 \times g$, $4^{\circ} \mathrm{C}$, for $10 \mathrm{~min}$, unconjugated antibodies were collected and applied to assess the coupling efficiency of the europium microspheres to the antibody. The conjugates were dissolved again and blocked in $1 \mathrm{~mL}$ of $1 \%$ BSA to quench the unlabeled groups for $15 \mathrm{~min}$, and then the centrifugation process was repeated. Finally, the conjugates were resuspended in $400 \mu \mathrm{L}$ of PBS-Tween buffer and stored at $4^{\circ} \mathrm{C}$ for further experiments.

\section{Establishment of CTRFIA for the Detection of Streptomycin in Milk}

Fabrication of Lateral Flow Test Strip. A lateral flow test strip consisted of a sample pad, an absorption pad, an adhesive backing card, and an NC membrane. The sample pad was immersed in 0.01 $M$ PBS containing $1 \%$ BSA, $5 \%$ sucrose, and $0.5 \%$ Tween-20 for 1 $\mathrm{h}$ in advance, followed by drying at $37^{\circ} \mathrm{C}$ overnight. Then, STR-OVA and goat anti-mouse antibodies were dispensed onto the NC membrane using a BioDotXYZ3060 dispensing platform (BioDot Inc., Irvine, $\mathrm{CA})$ to serve as the test (T) line and control (C) line, respectively, at a distance of $4 \mathrm{~mm}$. The lateral flow test strips were assembled as follows: briefly, the NC membrane lined with coating antigens was pasted onto the center of plastic backing card and the pretreated sample pad was pasted with one end partly covering
$(2 \mathrm{~mm})$ the NC membrane. Next, the absorbent pad was pasted onto the other side of the backing card also overlying $(2 \mathrm{~mm})$ the $\mathrm{NC}$ membrane. Then, the whole plate was cut into 4-mm-wide strips using a CM4000 guillotine (BioDot Inc.) and sealed in an aluminum foil bag with desiccant.

CTRFIA Protocol for Streptomycin Detection. A micro-well format of lateral flow analysis was performed. The principle of the immunoassay procedure was based on competitive binding between streptomycin in samples and coating antigens with the probes. In CTRFIA, the detection probes and $150 \mu \mathrm{L}$ of sample solution were added into microwells and mixed thoroughly. After 3 min, a test strip was vertically inserted into the well so that the liquid migrated up the NC membrane due to capillary force. The result can be judged qualitatively by the naked eye under UV light and quantitatively using a time-resolved fluorescence reader (Weice Biotechnology Co. Ltd.) after $10 \mathrm{~min}$. If there is no streptomycin residue in a sample, the STR-mAb probes are captured by coating antigens immobilized on the test line, resulting in strong fluorescence signal intensity. If streptomycin is present in a sample, the probes will conjugate with streptomycin, and the intensity of the $\mathrm{T}$ line decreases as the amount of streptomycin in the sample increases (Figure 2).

Results were expressed as means \pm standard deviation (SD). Data analysis was performed with Origin 2018 (OriginLab, Northampton, MA), GraphPad Prism 8.0 (GraphPad Software Inc., San Diego, CA).

\section{RESULTS AND DISCUSSION}

\section{Evaluation of Europium Microspheres}

The photological properties of europium microspheres are shown in Figure 3. The maximum absorption peak was around $340 \mathrm{~nm}$ and the emission wavelength was $615 \mathrm{~nm}$. We studied the effects of different buffers on the fluorescence and storage stability of europium microspheres by measuring the fluorescence spectra of europium microspheres in different buffers over time. Figure 4 shows that europium microspheres exhibited high fluorescence intensity in PBS buffer (PBS 0.01 $M$, $\mathrm{pH}$ 8.0), which was then chosen as the reaction buffer for subsequent experiments. To estimate the capacity of europium microspheres to couple with antibodies, 100 $\mu \mathrm{L}$ of a solution of europium microspheres was reacted with $25,50,100,200,400$, and $800 \mu \mathrm{g}$ of anti-STR mAb, and the BCA Protein Assay Kit (Pierce/ThermoFisher Scientific Inc., Waltham, MA) was used to measure the amount of anti-STR mAb in supernatant after coupling and centrifugation. The maximum coupling amount was determined using the following equation: amount 

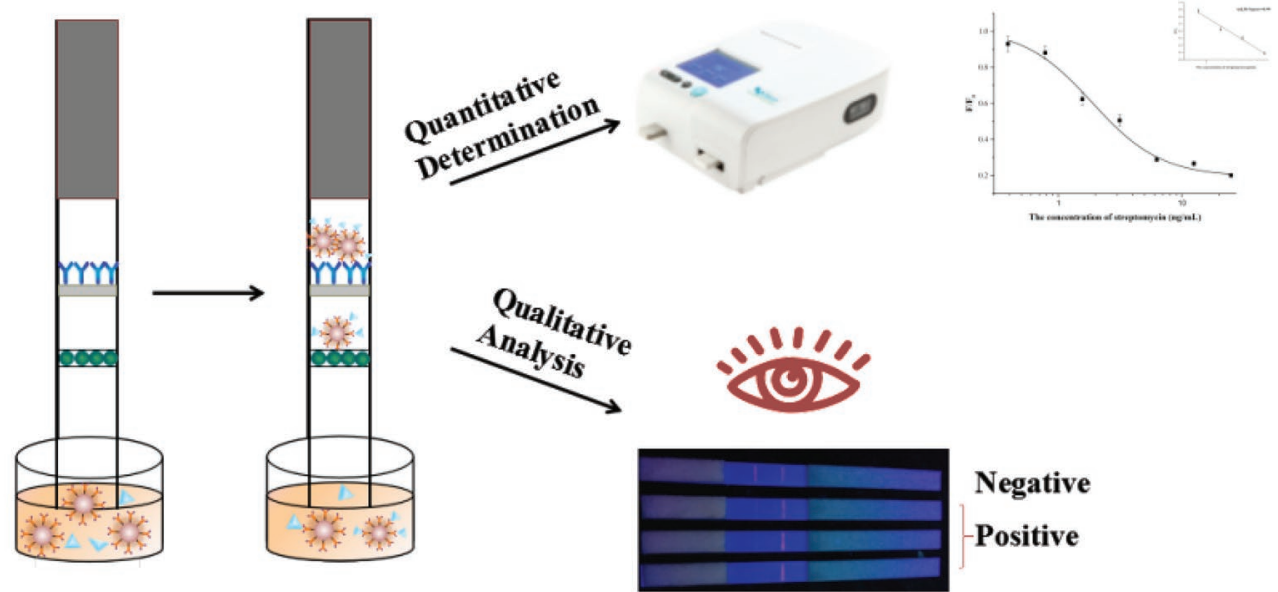

Negative

Positive

\section{- STR-OVA}

\section{Goat anti-mouse secondary antibody}

\section{Europium microsphere- antibody conjugate}

Figure 2. Schematic illustration of chromatographic time-resolved fluoroimmunoassay (CTRFIA) for qualitative and quantitative determination of streptomycin in milk. STR-OVA = streptomycin-ovalbumin.

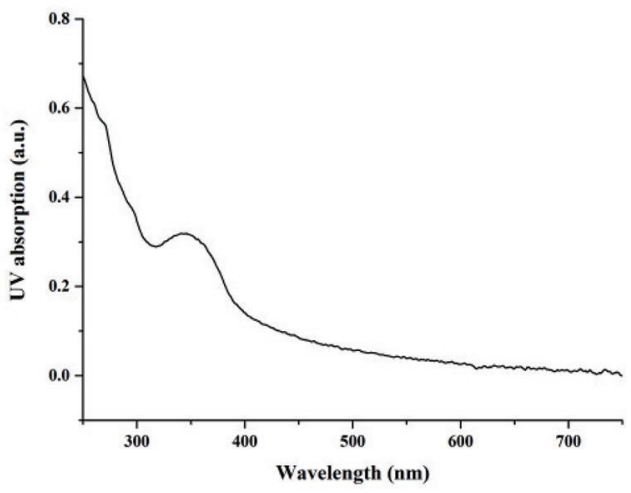

b

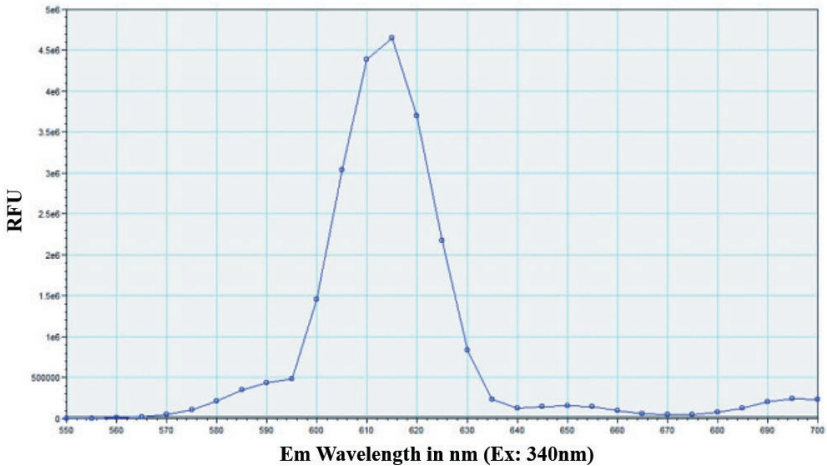

Figure 3. The absorption (a) and fluorescence spectra (b; in relative fluorescence units, RFU) of europium microspheres. Em $=$ emission: $\mathrm{Ex}=$ excitation.
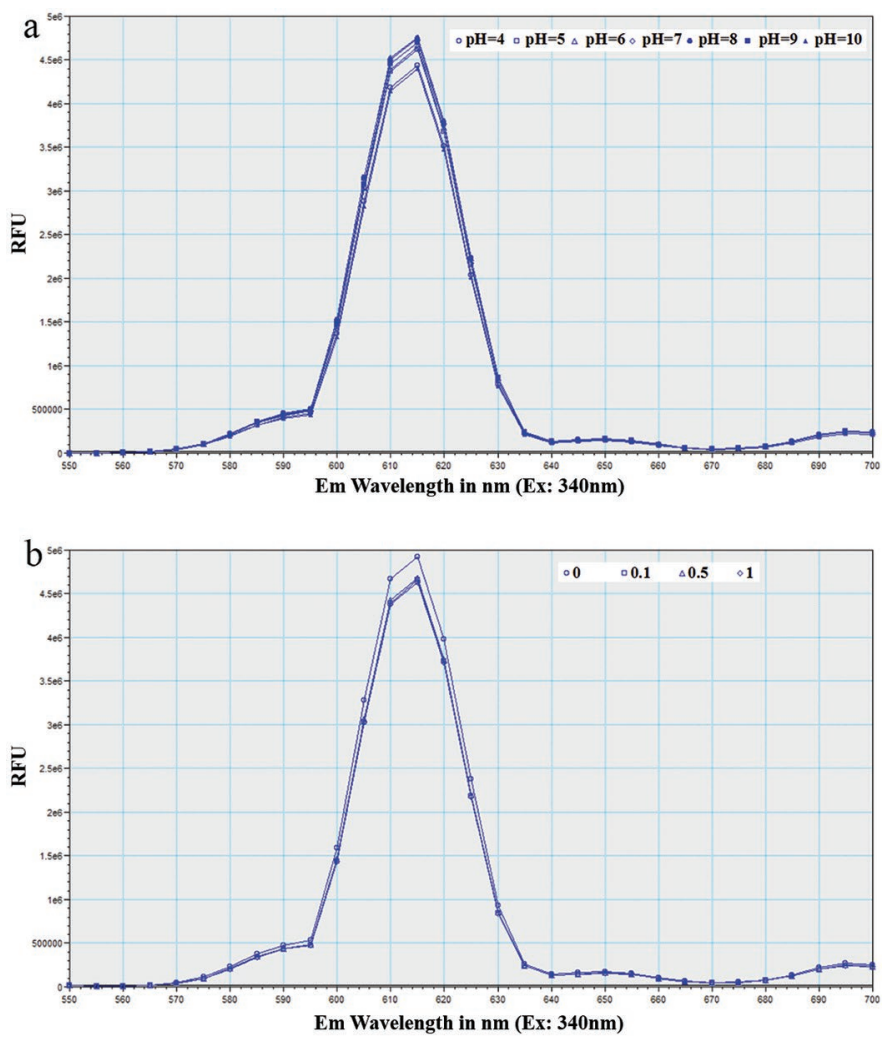

Figure 4. Effect of different solutions on the fluorescent property of europium microspheres (measured in relative fluorescence units, RFU): (a) $\mathrm{pH} 4$ to 10; (b) concentrations of $\mathrm{NaCl}$ from 0 to $1 \mathrm{M}$. 
of coupling antibody $(\mu \mathrm{g})=$ amount of antibody added $(\mu \mathrm{g})$ - concentration of supernatant protein $(\mu \mathrm{g} / \mathrm{mL})$ $\times$ volume $(\mathrm{mL})$. In Figure 5, the amount of coupling increased gradually with increasing addition of antibody and reached saturation at $170 \mu \mathrm{g}$.

\section{Optimization of CTRFIA Conditions}

The analytical performance of the immunochromatography strips can be affected by a number of variables, including the amount of anti-STR mAb and coating antigens on $\mathrm{T}$ line, the volume of fluorescence probes, the quantity of EDC, and the reaction time. To optimize these variables, we analyzed the fluorescence intensity and inhibition ratio of control (negative, $0 \mathrm{ng} /$ $\mathrm{mL}$ ) and spiked samples $(12.5 \mathrm{ng} / \mathrm{mL})$. Each value was based on 3 measurements. The inhibition ratio was calculated by the following formula: Inhibition ratio $=1$ $-\mathrm{F} / \mathrm{F}_{0}$, where $\mathrm{F}$ and $\mathrm{F}_{0}$ are defined as the fluorescence intensity of control and spiked samples, respectively.

\section{Orthogonal Optimization}

An orthogonal L9 $(3)^{3}$ experiment was performed to optimize the amount of anti-STR mAb and antigens and the volume of fluorescence probes. The results are shown in Figure 6 and Table 1. Test strips achieved better fluorescence intensity and inhibition rate when the concentration of STR-OVA dispersed on the test line was $0.5 \mathrm{mg} / \mathrm{mL}$ and $2 \mu \mathrm{L}$ of fluorescent probe was added. To decrease the consumption of reagents while

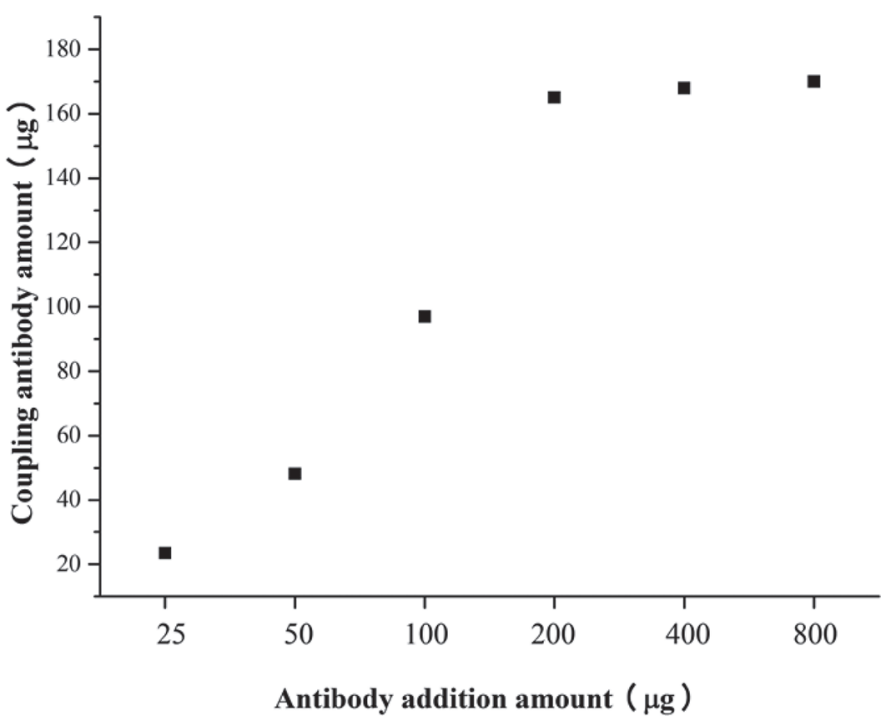

Figure 5. Effect of adding different amounts of antibody on coupling with europium microspheres.
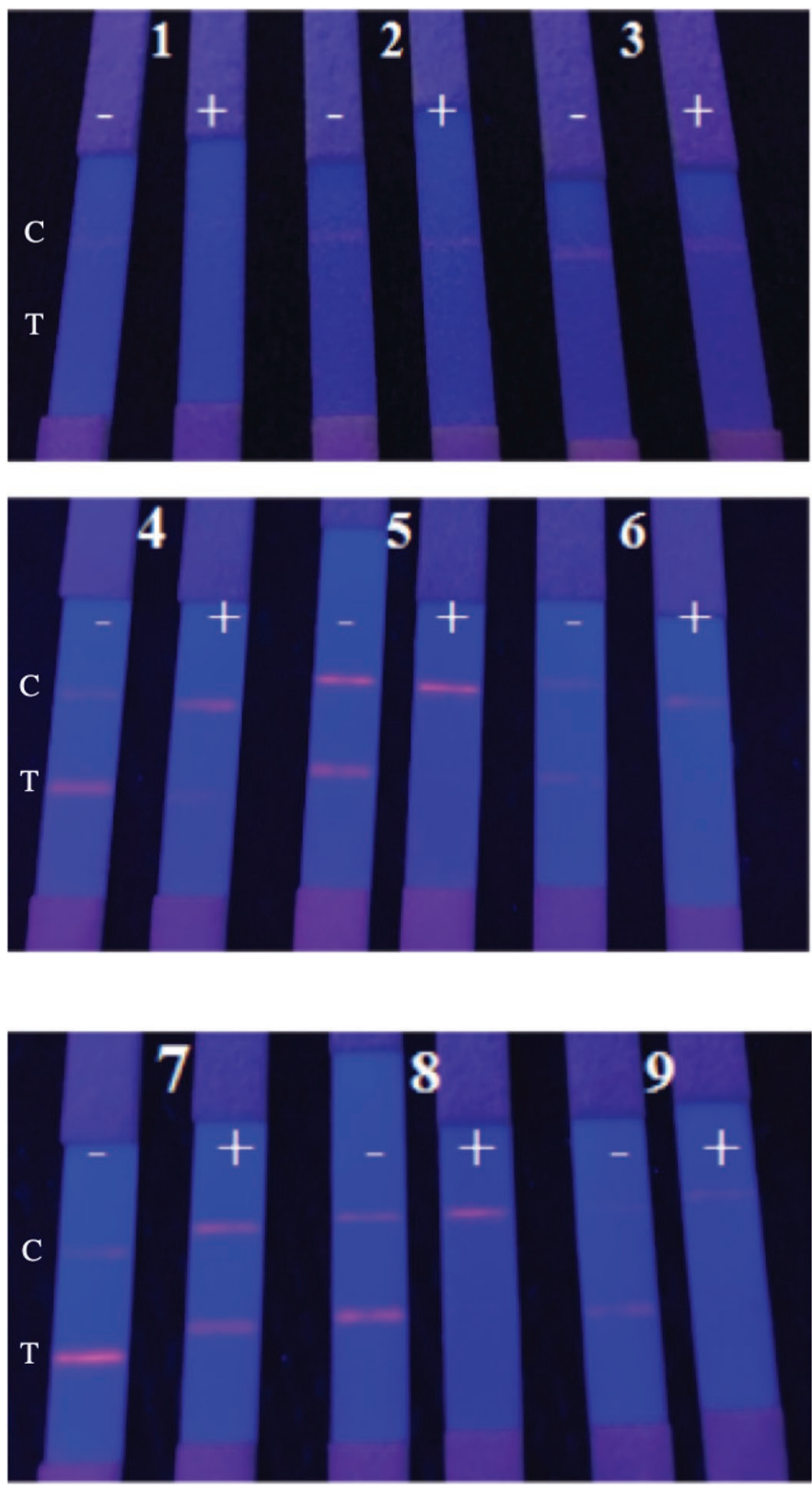

Figure 6. Immunochromatography of orthogonal L9 $(3)^{3}$ test to optimize the amount of anti-streptomycin $\mathrm{mAb}$ and antigens and the volume of fluorescence probes. Lane numbers represent sample numbers; - and + represent negative and positive samples, respectively; $\mathrm{C}$ $=$ control line; and $\mathrm{T}=$ test line

achieving high sensitivity, we found that $2 \mu \mathrm{L}$ of fluorescent probe prepared by coupling $5 \mu \mathrm{g}$ of anti-STR $\mathrm{mAb}$ with $10 \mu \mathrm{L}$ of europium microspheres was satisfactory.

\section{Optimization of the Amount of EDC}

The fluorescence probes were prepared by conjugating europium microspheres with antibodies through a 
Table 1. The results of chromatographic orthogonal L9 $(3)^{3}$ test to optimize the amount of anti-streptomycin (STR) mAb and antigens (streptomycin-ovalbumin, STR-OVA) and the volume of fluorescence probes (europium-antibody conjugate)

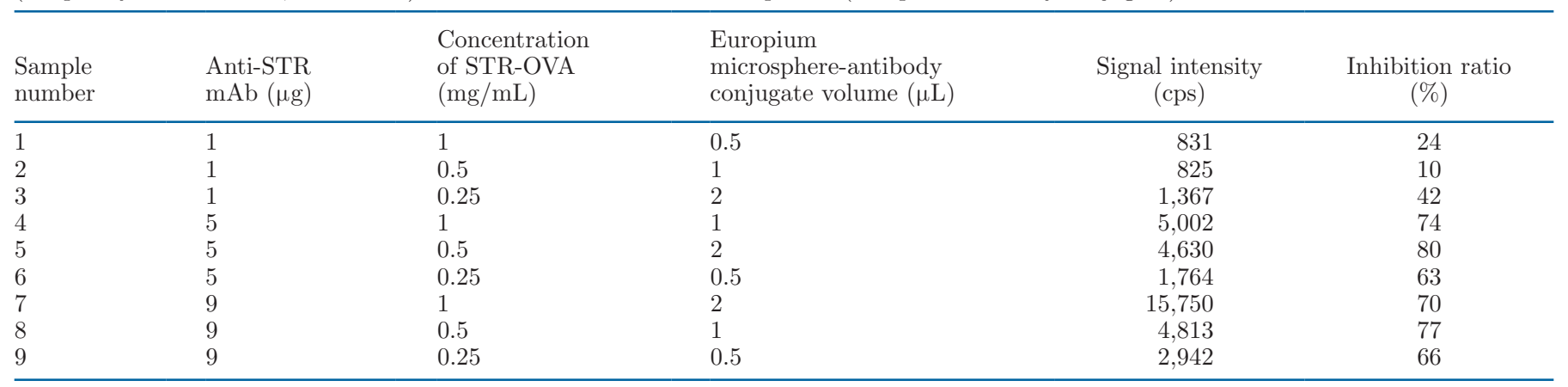

reaction between carboxyl groups on europium microspheres and amino groups on antibodies. The effect of EDC/NHS catalyst on the covalent coupling reaction is shown in Figure 7. Acyl-isocyanurate was synthesized when EDC activated the carboxylic groups on the surface of microspheres; NHS can both protect unstable acyl-isocyanurate in aqueous solution and accelerate the conjugation reaction with antibody amino to form carboxyfluorescein diacetate succinimidyl ester (CFSE). In addition, to promote the coupling reaction, the conjugation procedure should be conducted in an acidic solution, in which the positively charged antibodies and negatively charged europium microspheres are attracted electronically.

The dose of EDC (from 1 to $40 \mu \mathrm{g}$ ) was optimized and the results are shown in Figure 8. The fluorescence signal intensity and inhibition ratio peaked when $10 \mu \mathrm{g}$ of EDC was added; when this amount was exceeded, fluorescence intensity was stable but the inhibition ratio decreased sharply. Because excess catalyst would suppress the immunoreaction by aggravating homocou- pling and blocking active sites of antibody, $10 \mu \mathrm{g}$ of EDC was chosen as the optimal amount.

\section{Optimization of Immunoreaction Time}

Immunoreaction time is a key factor. On the one hand, a prolonged response time will promote a better combination between the fluorescence probes and antigens to achieve high fluorescence intensity. On the other hand, it also may lead to false-negative test results. As shown in Figure 9, the maximum competitive inhibition ratio was observed at $3 \mathrm{~min}$ and the intensity of the $\mathrm{T}$ line signal of a negative sample increased gradually and remained stable at $20 \mathrm{~min}$. Thus, $3 \mathrm{~min}$ was selected as the optimal immunoreaction time.

\section{Evaluation of CTRFIA}

As shown in Figure 10, as the concentration of streptomycin increased, fluorescence intensities of $\mathrm{T}$ lines decreased. The visible fluorescence signal of the
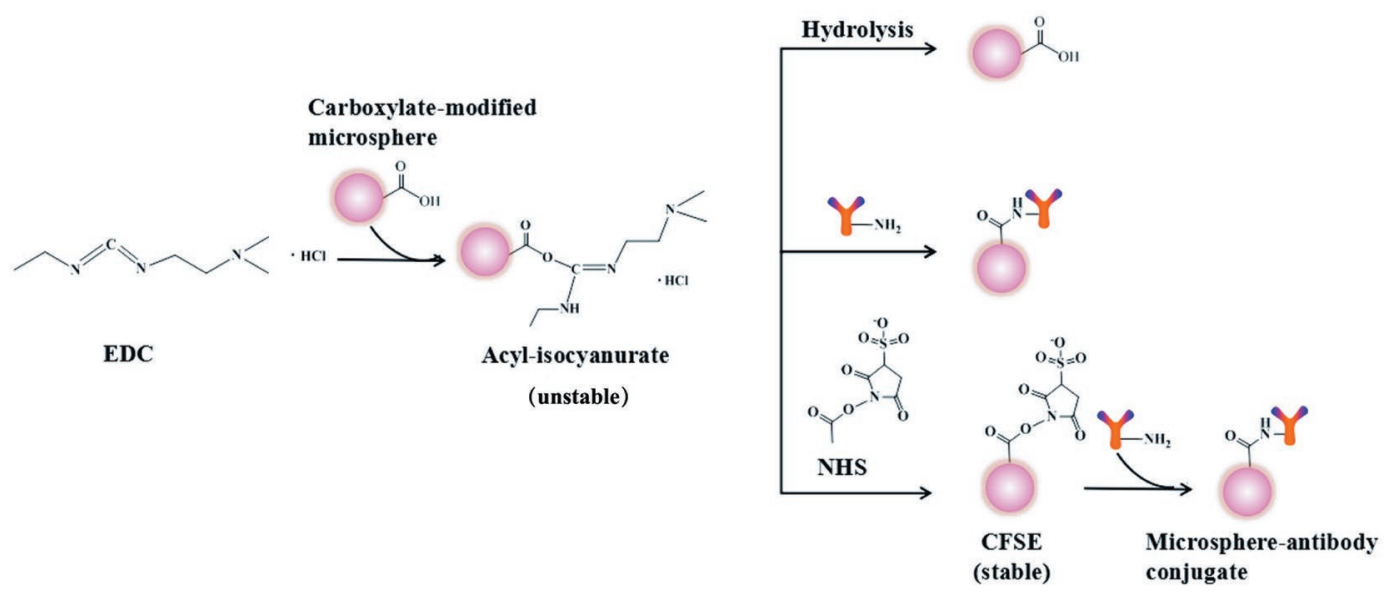

Figure 7. The mechanism of the coupling reaction between europium microsphere and antibody by 1-ethyl-3-(3-dimethylaminopropyl)carbodiimide (EDC) and hydroxysuccinimide (NHS). CFSE = carboxyfluorescein diacetate succinimidyl ester. 


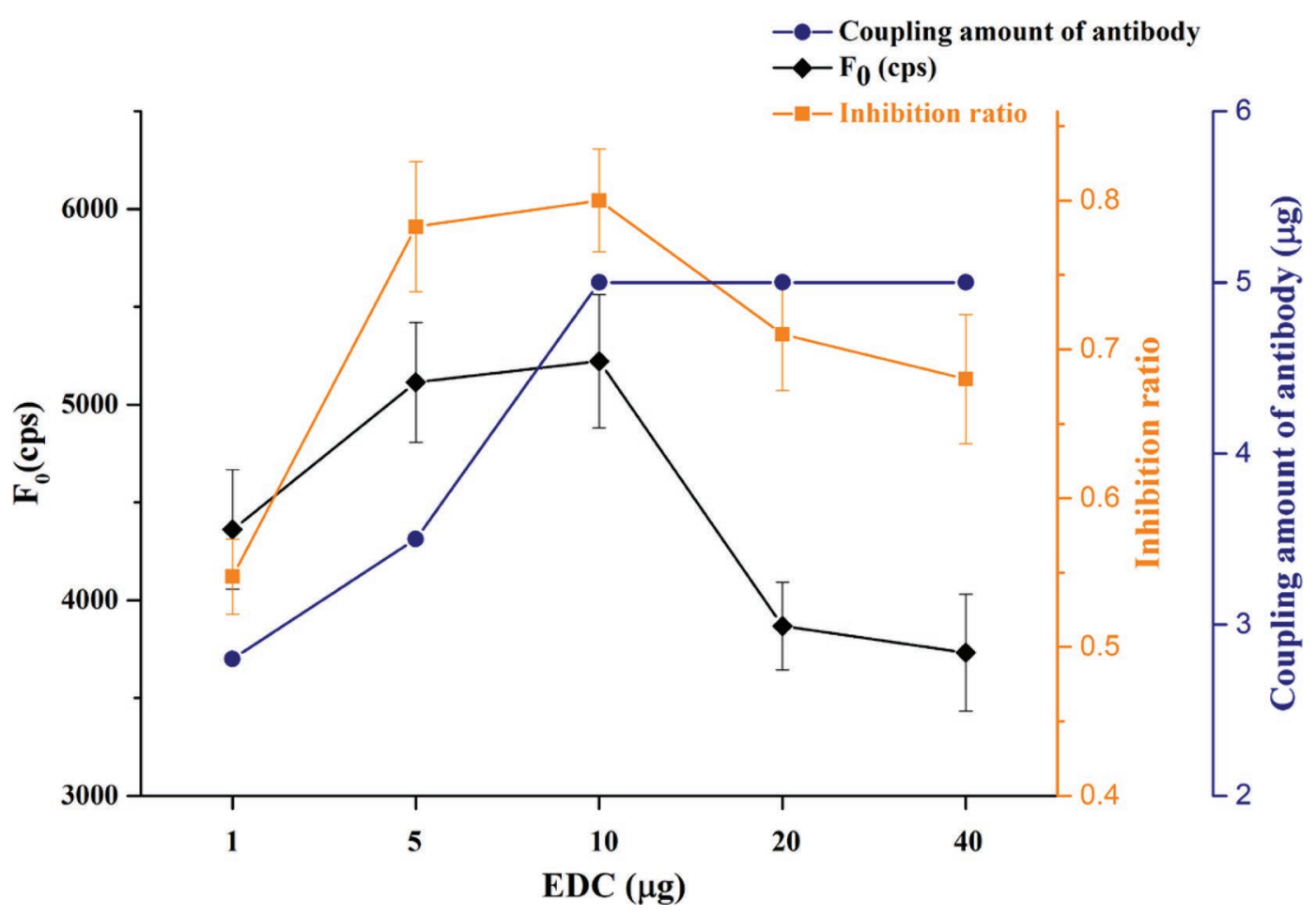

Figure 8. Effect of the amount of 1-ethyl-3-(3-dimethylaminopropyl)-carbodiimide (EDC) on performance of chromatographic time-resolved fluoroimmunoassay during the antibody coupling procedure. Inhibition ratio $=1-\mathrm{F} / \mathrm{F}_{0}$, where $\mathrm{F}$ and $\mathrm{F}_{0}$ represent the fluorescence intensity of control and spiked samples, respectively. Error bars represent SD.

T line disappeared at $6.25 \mathrm{ng} / \mathrm{mL}$, which was therefore defined as the cutoff value of CTRFIA. Calibration curves were then constructed by plotting the ratios

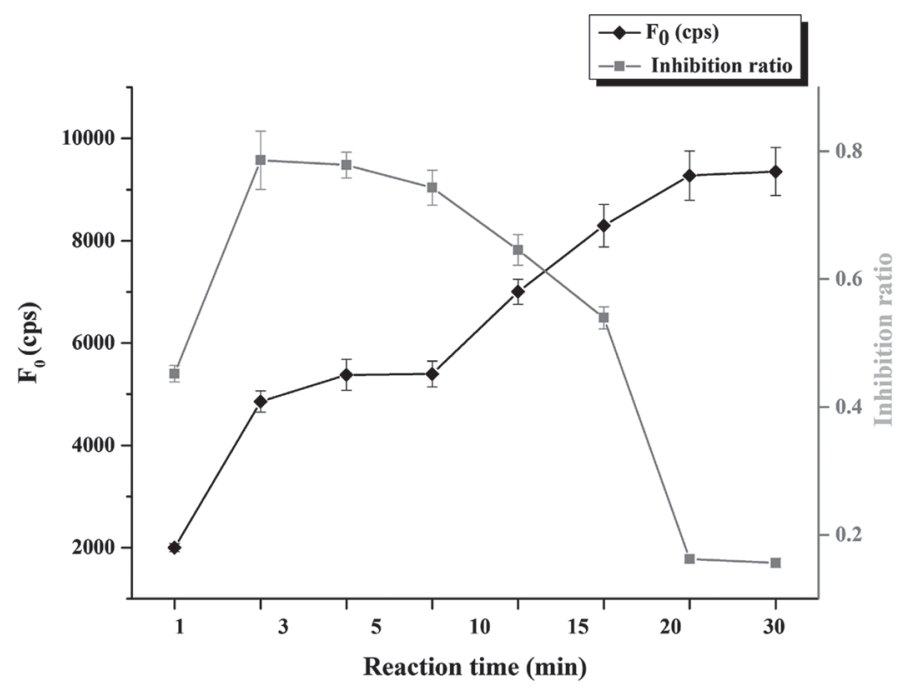

Figure 9. Influence of reaction time on chromatographic timeresolved fluoroimmunoassay performance. Inhibition ratio $=1-\mathrm{F}$ / $\mathrm{F}_{0}$, where $\mathrm{F}$ and $\mathrm{F}_{0}$ represent the fluorescence intensity of control and spiked samples, respectively. Error bars represent SD. between fluorescence intensities of spiked and blank samples $\left(\mathrm{B} / \mathrm{B}_{0}\right)$ against the logarithm concentrations of analytes (Figure 11). The half-maximal inhibitory concentration $\left(\mathrm{IC}_{50}\right)$ for streptomycin was $2.62 \mathrm{ng} / \mathrm{mL}$ and the within-assay coefficient of variation $(\mathbf{C V})$ was $<6.2 \%$. A satisfactory linear range was from 0.8 to 6.25 $\mathrm{ng} / \mathrm{mL}$ with the coefficient of determination $\left(\mathbf{R}^{2}\right)$ being $>0.99$. The limit of detection, defined as the concentration corresponding to the mean value of the 20 buffer blank values plus 3 times the $\mathrm{SD}$, was $0.58 \mathrm{ng} / \mathrm{mL}$.

Spiked recovery experiments were carried out to evaluate the accuracy and precision of the method. Different concentrations of streptomycin were added to blank raw milk samples. At spiked concentrations of 1 to 4 $\mathrm{ng} / \mathrm{mL}$, mean recoveries of streptomycin ranged from 85.6 to $108.3 \%$ (Table 2), within the allowable range of 80 to $110 \%$, and the CV were $<12.1 \%$. This indicated that the proposed method meets streptomycin residue detection requirements in milk samples.

The specificity of the method was estimated by calculating cross-reactivity with 9 antibiotics. As illustrated in Table 3, there was negligible cross-reactivity $(<0.1 \%)$ with other compounds except dihydrostreptomycin (126.5\%). The immunoreaction thus demonstrated high specificity for detecting streptomycin and dihydrostreptomycin. 


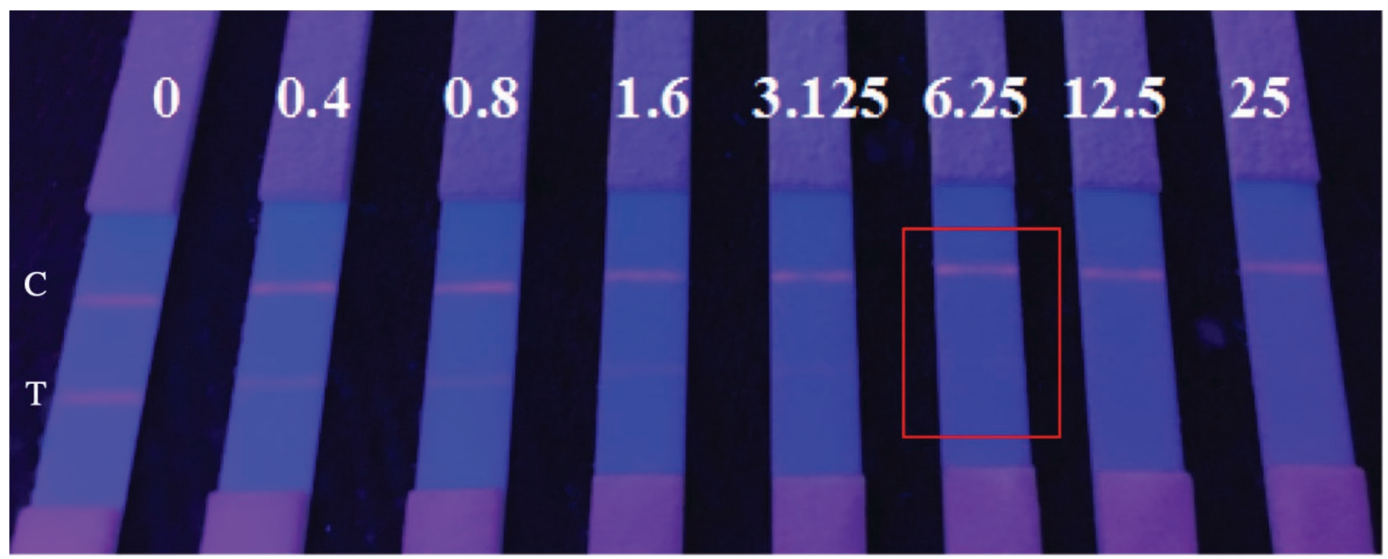

Figure 10. Cutoff value of chromatographic time-resolved fluoroimmunoassay (CTRFIA) for streptomycin in milk. Numbers in this figure represent different concentrations of streptomycin; the red box indicates the complete disappearance of the test line; $\mathrm{C}=$ control line; and $\mathrm{T}=$ test line.

The stability of the assay was evaluated by an accelerated aging test using the same batch of strips after storage at $60^{\circ} \mathrm{C}$ for $7 \mathrm{~d}$. The fluorescence intensity of control and spiked samples was observed daily. As shown in Figure 12, we observed no significant change in variables in the first $4 \mathrm{~d}$. According to the Arrhenius equation, the test strips could be stored at room temperature for at least 12 mo.

\section{Determination of Streptomycin in Milk}

The use of CTRFIA detection in milk was evaluated under optimal conditions. We analyzed streptomycin in 20 randomly selected milk samples collected from several dairy farms in Hebei Province of China and compared the results with those obtained using ultrahigh-performance liquid chromatography-tandem MS (UHPLC-MS/MS; Gremilogianni et al., 2010). As shown in Table 4, streptomycin was detected in 13 samples at concentrations ranging from 1.84 to 13.37 $\mu \mathrm{g} / \mathrm{kg}$ by CTRFIA, and in 13 samples at concentrations ranging from 2.18 to $12.7 \mu \mathrm{g} / \mathrm{kg}$ by UHPLC-MS/ MS. The significant correlation between values obtained from CTRFIA and UHPLC-MS/MS (Figure 13) showed that CTRFIA was a reliable method that can be applied to measure streptomycin concentrations in real samples.

Many reports of immunoassays applied to sensitive drug residue detection in milk have been published. The most commonly used methods are ELISA and colloidal gold immunochromatographic assay (GICA). We found that the sensitivity and simplicity of CTRFIA were better than those of other methods (Table 5). The CTRFIA method reported here has the advantages that large europium chelates in a single europium microsphere amplify the fluorescent signal and that covalent binding between antibody and label is more stable than electrostatic adsorption, which helps reduce
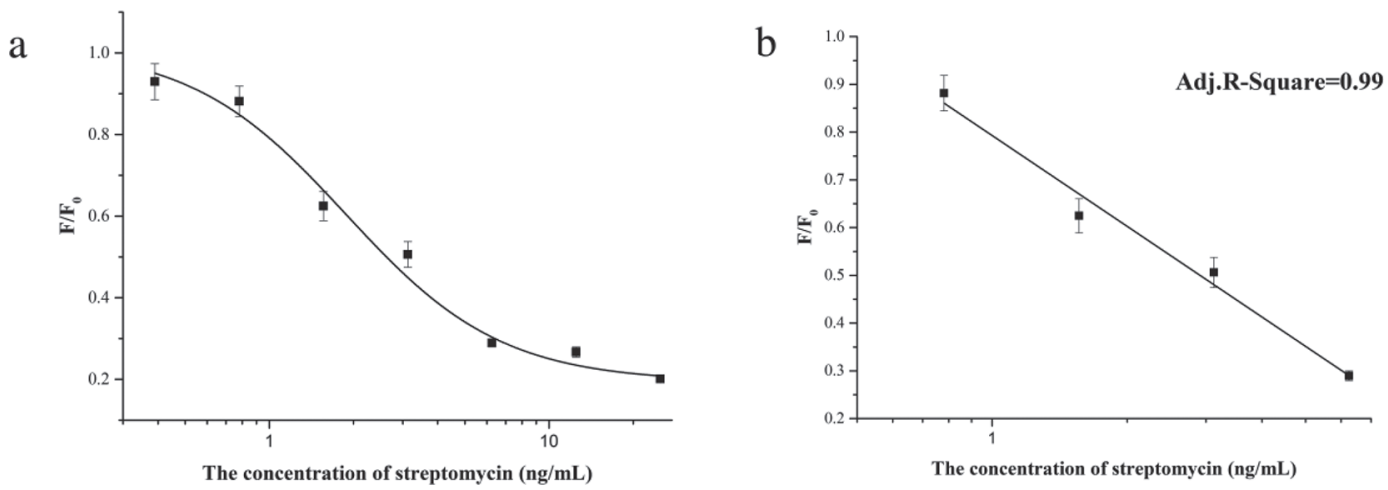

Figure 11. Standard curve (a) and linear range (b) of chromatographic time-resolved fluoroimmunoassay (CTRFIA) for streptomycin in milk. Error bars represent SD. F and $\mathrm{F}_{0}$ represent the fluorescence intensity of control and spiked samples, respectively. 
Table 2. Recoveries of streptomycin in spiked raw milk samples by chromatographic time-resolved fluoroimmunoassay

\begin{tabular}{lccr}
\hline $\begin{array}{l}\text { Spiked } \\
(\mathrm{ng} / \mathrm{mL})\end{array}$ & $\begin{array}{c}\text { Measure }^{1} \\
(\mathrm{ng} / \mathrm{mL})\end{array}$ & $\begin{array}{c}\text { Recovery } \\
(\%)\end{array}$ & $\begin{array}{c}\mathrm{CV} \\
(\%)\end{array}$ \\
\hline 1 & $0.91 \pm 0.08$ & 91 & 8.7 \\
2 & $2.16 \pm 0.26$ & 108.3 & 12.1 \\
4 & $3.42 \pm 0.18$ & 85.6 & 5.3 \\
\hline
\end{tabular}

${ }^{1}$ Mean \pm SD $(\mathrm{n}=3)$.

Table 3. Specificity evaluation of chromatographic time-resolved fluoroimmunoassay

\begin{tabular}{lcc}
\hline Compound & $\begin{array}{c}\mathrm{IC}_{50}{ }^{1} \\
(\mathrm{ng} / \mathrm{mL})\end{array}$ & $\begin{array}{c}\text { Cross } \\
\text { reactivity }(\%)\end{array}$ \\
\hline Streptomycin & 2.62 & 100 \\
Dihydrostreptomycin & 2.07 & 126.5 \\
Gentamycin & $>3,000$ & $<0.1$ \\
Spectinomycin & $>3000$ & $<0.1$ \\
Kanamycin & $>3,000$ & $<0.1$ \\
Tobramycin & $>3,000$ & $<0.1$ \\
Amikacin & $>3,000$ & $<0.1$ \\
Sulfadimidine & $>3,000$ & $<0.1$ \\
Tetracycline & $>3,000$ & $<0.1$ \\
Chloramphenicol & $>3,000$ & $<0.1$ \\
\hline
\end{tabular}

${ }^{1}$ Half-maximal inhibitory dose.

Table 4. Determination of streptomycin residue in milk samples by chromatographic time-resolved fluoroimmunoassay (CTRFIA) and ultra-high-performance liquid chromatography-tandem MS (UHPLCMS/MS) ${ }^{1}$

\begin{tabular}{lcc}
\hline Sample no. & CTRFIA & UHPLC-MS/MS \\
\hline 1 & 2.74 & 2.35 \\
2 & 4.35 & 3.99 \\
3 & $\mathrm{ND}^{2}$ & $\mathrm{ND}$ \\
4 & $\mathrm{ND}$ & $\mathrm{ND}$ \\
5 & $\mathrm{ND}$ & $\mathrm{ND}$ \\
6 & $\mathrm{ND}$ & 13.37 \\
7 & 12.7 & 2.90 \\
8 & 3.35 & $\mathrm{ND}$ \\
9 & $\mathrm{ND}$ & 2.19 \\
10 & 2.37 & 4.16 \\
11 & 4.28 & $\mathrm{ND}$ \\
12 & $\mathrm{ND}$ & 3.10 \\
13 & 3.69 & $\mathrm{ND}$ \\
14 & $\mathrm{ND}$ & 2.90 \\
15 & 3.14 & 2.55 \\
16 & 2.71 & 2.19 \\
17 & 2.57 & 1.84 \\
18 & 2.18 & $\mathrm{ND}$ \\
19 & $\mathrm{ND}$ & 2.54 \\
20 & 3.25 & \\
\hline
\end{tabular}

${ }^{1}$ Each result was carried out in 3 replicates. See Figure 13 for data correlation analysis.

${ }^{2}$ Not detected.

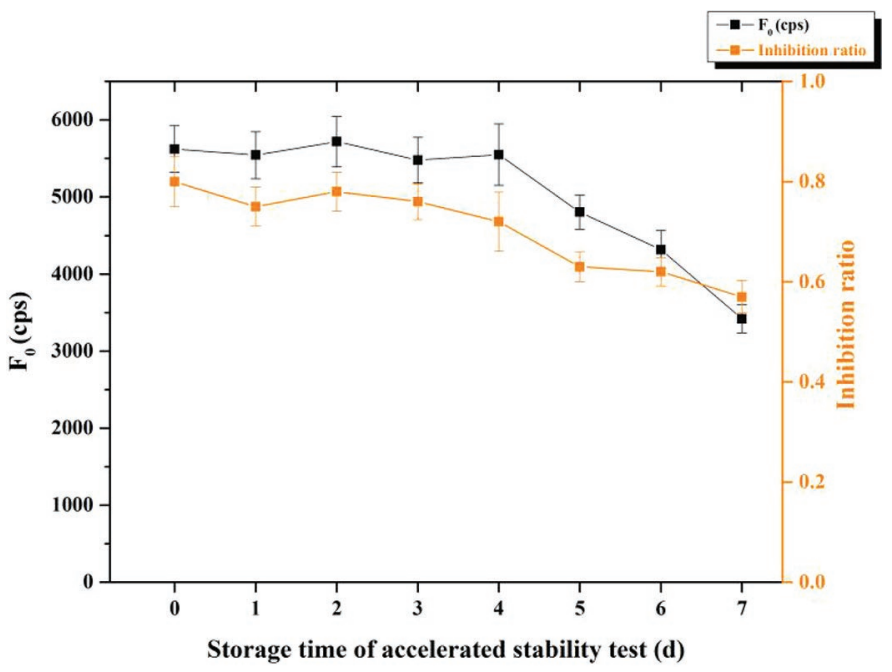

Figure 12. Evaluation of chromatographic time-resolved fluoroimmunoassay (CTRFIA) stability. Inhibition ratio $=1-\mathrm{F} / \mathrm{F}_{0}$, where $\mathrm{F}$ and $\mathrm{F}_{0}$ are defined as the fluorescence intensity of control and spiked samples, respectively. Error bars represent SD.

matrix interference. Similar results have been observed in previous studies (Wang et al., 2016).

\section{CONCLUSIONS}

The proposed CTRFIA method performed well for detection of streptomycin residues in milk with a low detection limit and acceptable stability, reproducibility, and accuracy. The convenient operation without the

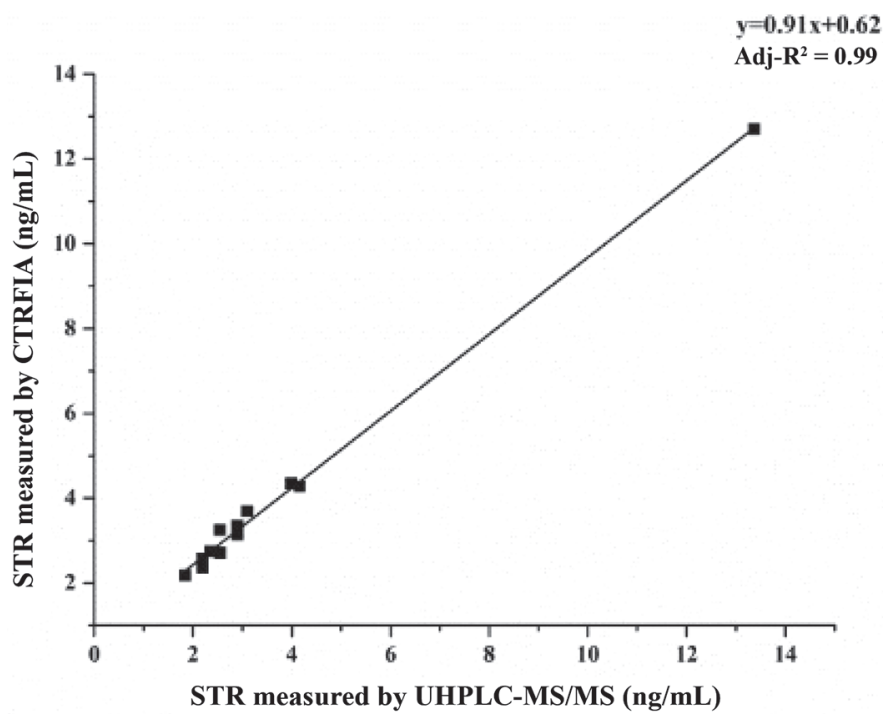

Figure 13. Correlation between chromatographic time-resolved fluoroimmunoassay (CTRFIA) and ultra-high-performance liquid chromatography-tandem MS (UHPLC-MS/MS) for streptomycin (STR) determination in real milk samples. 


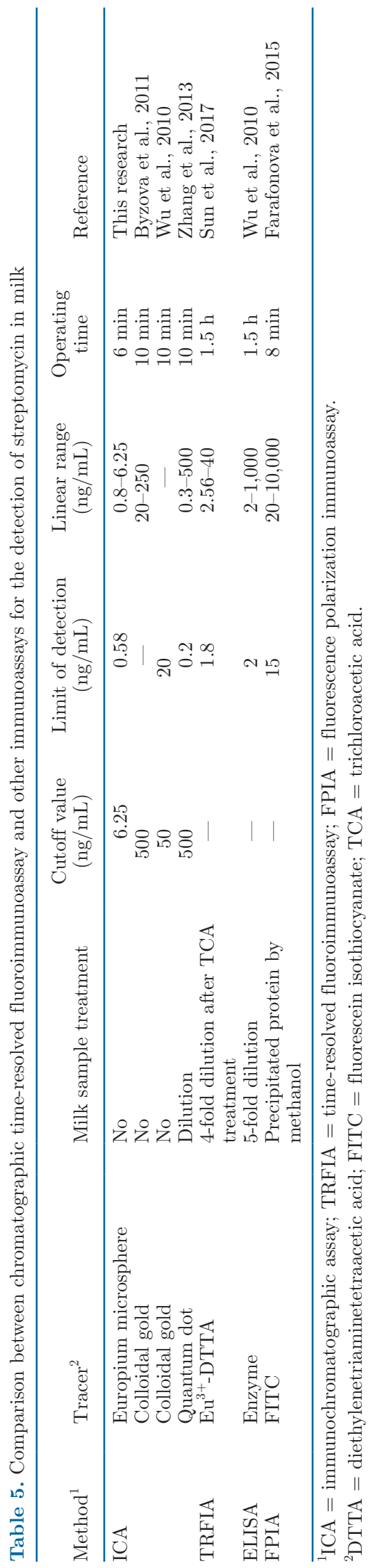

need for sample pretreatment, the extremely short detection time, and the high sensitivity of the proposed method indicate that it has potential in food safety screening.

\section{ACKNOWLEDGMENTS}

This study was financed by the National Natural Science Foundation of China (31672600) and Sanming Project of Medicine in Shenzhen (SZSM201611068). The authors declare that they have no conflicts of interest.

\section{REFERENCES}

Buenzli, J.-C. G. 2010. Lanthanide luminescence for biomedical analyses and imaging. Chem. Rev. 110:2729-2755. https://doi.org/10 $.1021 / \mathrm{cr} 900362 \mathrm{e}$.

Byzova, N. A., E. A. Zvereva, A. V. Zherdev, S. A. Eremin, P. G. Sveshnikov, and B. Dzantiev. 2011. Pretreatment-free immunochromatographic assay for the detection of streptomycin and its application to the control of milk and dairy products. Anal. Chim. Acta 701:209-217. https://doi.org/10.1016/j.aca.2011.06.001.

Chafer-Pericas, C., A. Maquieira, and R. Puchades. 2010. Fast screening methods to detect antibiotic residues in food samples. TrAC Trends Analyt. Chem. 29:1038-1049. https://doi.org/10.1016/j trac.2010.06.004

Chen, B., S. Saghaian, and Y. Zheng. 2018. Organic labelling, private label, and U.S. household demand for fluid milk. Appl. Econ. 50:3039-3050. https://doi.org/10.1080/00036846.2017.1414936.

Cho, I.-H., A. Bhunia, and J. Irudayaraj. 2015. Rapid pathogen detection by lateral-flow immunochromatographic assay with gold nanoparticle-assisted enzyme signal amplification. Int. J. Food Microbiol. 206:60-66. https://doi.org/10.1016/j.ijfoodmicro.2015 .04 .032 .

Farafonova, O. V., S. V. Vasiliev, S. A. Eremin, and T. N. Ermolaeva. 2015. Determination of aminoglycosides in food by fluorescence polarization immunoassay. Int. Res. J. 7:65.

Gremilogianni, A. M., N. C. Megoulas, and M. A. Koupparis. 2010. Hydrophilic interaction vsion pair liquid chromatography for the determination of streptomycin and dihydrostreptomycin residues in milk based on mass spectrometric detection. J. Chromatogr. A 1217:6646-6651. https://doi.org/10.1016/j.chroma.2010.04.059.

Hagan, A. K., and T. Zuchner. 2011. Lanthanide-based time-resolved luminescence immunoassays. J. Anal. Bioanal. Chem. 400:28472864. https://doi.org/10.1007/s00216-011-5047-7.

Hu, L.-M., K. Luo, J. Xia, G.-M. Xu, C.-H. Wu, J.-J. Han, G.-G. Zhang, M. Liu, and W.-H. Lai. 2017. Advantages of time-resolved fluorescent nanobeads compared with fluorescent submicrospheres, quantum dots, and colloidal gold as label in lateral flow assays for detection of ractopamine. Biosens. Bioelectron. 91:95-103. https:/ /doi.org/10.1016/j.bios.2016.12.030.

Huang, X., Z. P. Aguilar, H. Xu, W. Lai, and Y. Xiong. 2016. Membrane-based lateral flow immunochromatographic strip with nanoparticles as reporters for detection: A review. Biosens. Bioelectron. 75:166-180. https://doi.org/10.1016/j.bios.2015.08.032.

Juntunen, E., T. Myyryläinen, T. Salminen, T. Soukka, and K. Pettersson. 2012. Performance of fluorescent europium(III) nanoparticles and colloidal gold reporters in lateral flow bioaffinity assay. Anal. Biochem. 428:31-38. https://doi.org/10.1016/j.ab.2012.06 .005 .

Peng, T., F. S. Zhang, W. C. Yang, D. X. Li, Y. Chen, Y. H. Xiong, H. Wei, and W. H. Lai. 2014. Lateral-flow assay for rapid quantitative detection of clorprenaline residue in swine urine. J. Food Prot. 10:1824-1829. https://doi.org/10.4315/0362-028X.JFP-14-103.

Posthuma-Trumpie, G. A., J. H. Wichers, and M. Koets. 2012. Amorphous carbon nanoparticles: A versatile label for rapid diagnostic 
(immuno)assays. Anal. Bioanal. Chem. 402:593-600. https://doi .org/10.1007/s00216-011-5340-5.

Soukka, T., K. Antonen, H. Hrm, A.-M. Pelkkikangas, P. Huhtinen, and T. Lövgren. 2003. Highly sensitive immunoassay of free prostate-specific antigen in serum using europium (III) nanoparticle label technology. Clin. Chim. Acta 328:45-58.

Sun, Y., J. Xie, T. Peng, J. Wang, S. Xie, K. Yao, C. Wang, S. Sun, X. Xia, and H. Jiang. 2017. A new method based on time-resolved fluoroimmunoassay for the detection of streptomycin in milk. Food Anal. Methods 10:2262-2269. https://doi.org/10.1007/s12161-017 $-0797-2$.

Tao, X., J. Wang, Y. Xie, X. Zuo, F. Mo, S. Zhou, and H. Li. 2017. Dual-label chemiluminescence strategy for multiplexed immunoassay of 20 fluoroquinolones, $15 \beta$-lactams, 15 sulfonamides, and CAP in milk. Food Anal. Methods 10:3009-3022. https://doi.org/ 10.1007/s12161-017-0865-7.

Taranova, N. A., A. N. Berlina, A. V. Zherdev, and B. B. Dzantiev. 2015. 'Traffic light' immunochromatographic test based on multicolor quantum dots for the simultaneous detection of several antibiotics in milk. Biosens. Bioelectron. 63:255-261. https://doi.org/ 10.1016/j.bios.2014.07.049

Verheijen, R., I. K. Osswald, R. Dietrich, and W. Haasnoot. 2000 Development of a one step strip test for the detection of (dihydro) streptomycin residues in raw milk. Food Agric. Immunol. 12:3140. https://doi.org/10.1080/09540100099607.

Wang, C., X. Li, T. Peng, Z. Wang, K. Wen, and H. Jiang. 2017a. Latex bead and colloidal gold applied in a multiplex immunochromatographic assay for high-throughput detection of three classes of antibiotic residues in milk. Food Control 77:1-7. https://doi .org/10.1016/j.foodcont.2017.01.016

Wang, D., Z. Zhang, and P. Li. 2015. Europium nanospheres-based time-resolved fluorescence for rapid and ultrasensitive determination of total aflatoxin in feed. J. Agric. Food Chem. 63:1031310318. https://doi.org/10.1021/acs.jafc.5b03746.

Wang, G. P., I. Basu, L. A. Beyer, H. T. Wong, D. L. Swiderski, S. S. Gong, and Y. Raphael. 2017b. Severe streptomycin ototoxicity in the mouse utricle leads to a flat epithelium but the peripheral neural degeneration is delayed. Hear. Res. 355:33-41. https://doi .org/10.1016/j.heares.2017.09.004.

Wang, P., R. Wang, W. Zhang, X. Su, and H. Luo. 2016. Novel fabrication of immunochromatographic assay based on up conversion phosphors for sensitive detection of clenbuterol. Biosens. Bioelectron. 77:866-870. https://doi.org/10.1016/j.bios.2015.10.053.

Wu, J.-X., S.-E. Zhang, and X.-P. Zhou. 2010. Monoclonal antibodybased ELISA and colloidal gold-based immunochromatographic assay for streptomycin residue detection in milk and swine urine.
J. Zhejiang Univ. Sci. B 11:52-60. https://doi.org/10.1631/jzus B0900215.

Xi, X., M. Zhang, M. Li, Y. Gong, M. Wang, Z. Chen, and W. Wang. 2013. Development of dcELISA method for rapid detection of streptomycin residue in milk and honey. J. Chin. Inst. Food Sci. Technol. 13:124-131.

Yu, F., S. Yu, and L. Yu. 2014. Determination of residual enrofloxacin in food samples by a sensitive method of chemiluminescence enzyme immunoassay. Food Chem. 149:71-75. https://doi.org/10 $.1016 / \mathrm{j}$.foodchem.2013.10.024.

Zeng, H., J. Chen, C. Zhang, X. Huang, Y. Sun, Z. Xu, and H. Lei. 2016. Broad-specificity chemiluminescence enzyme immunoassay for (fluoro)quinolones: Hapten design and molecular modeling study of antibody recognition. Anal. Chem. 88:3909-3916. https:/ /doi.org/10.1021/acs.analchem.6b00082.

Zhang, S., S. Piepers, R. Shan, L. Cai, S. Mao, J. Zou, T. Ali, S. De Vliegher, and B. Han. 2018. Phenotypic and genotypic characterization of antimicrobial resistance profiles in Streptococcus dysgalactiae isolated from bovine clinical mastitis in 5 provinces of China. J. Dairy Sci. 101:3344-3355. https://doi.org/10.3168/jds .2017-14031.

Zhang, Z., M. Lin, and S. Zhang. 2013. Detection of aflatoxin M1 in milk by dynamic light scattering coupled with superparamagnetic beads and gold nanoprobes. J. Agric. Food Chem. 61:4520-4525 https://doi.org/10.1021/jf400043z.

Zhang, Z., X. Tang, D. Wang, Q. Zhang, P. Li, and X. Ding. 2015. Rapid on-site sensing aflatoxin B1 in food and feed via a chromatographic time-resolved fluoroimmunoassay. PLoS One 10:e0123266. https://doi.org/10.1371/journal.pone.0123266.

Zhao, J., Y. Wu, H. Tao, H. Chen, W. Yang, and S. Qiu. 2017. Colorimetric detection of streptomycin in milk based on peroxidasemimicking catalytic activity of gold nanoparticles. RSC Advances 7:38471-38478. https://doi.org/10.1039/C7RA06434A.

\section{ORCIDS}

Zile Wang (๑) https://orcid.org/0000-0002-4513-688X Yuanze Sun () https://orcid.org/0000-0002-8384-7594

Demei Liang () https://orcid.org/0000-0002-2899-5113

Yuyang Zeng @ https://orcid.org/0000-0002-7707-0416

Shuang He () https://orcid.org/0000-0003-2391-4748

Tao Peng () https://orcid.org/0000-0002-2898-7586

Haiyang Jiang @ https://orcid.org/0000-0002-0529-0719 